\title{
PSYCHOLOGICAL ASPECTS OF ENSURING FLIGHT SAFETY IN CIVIL AVIATION
}

\author{
E.V. Luzik, A.N. Akmaldinova
}

National Aviation University, 1 Cosmonavta Komarova Ave, 03058 Kiev, Ukraine. Received 3009 2004, accepted 06032006

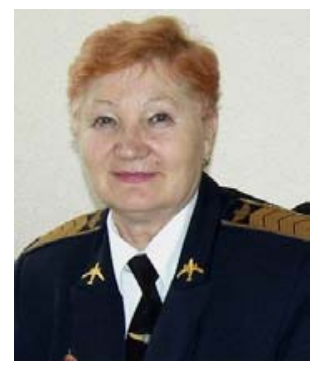

Elvira V. LUZIK, Prof Dr

Present position: Head of Pedagogy Department, National Aviation University (Kiev, Ukraine.)

Home tel.: (044) 5167855

Office tel.: (044) 4067123

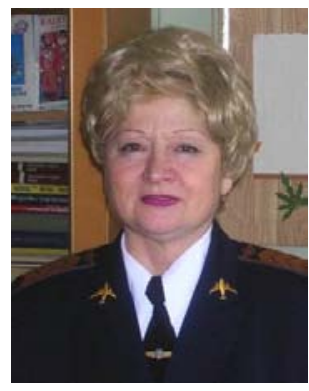

\section{Aleksandra N. AKMALDINOVA, Prof Ph D}

Present position: Head of the Foreign Languages for Special Purposes Department, National Aviation University (Kiev, Ukraine), responsible for corresponding with the Editorial Board.

Home tel.: (044) 4578037

Office tel.: (044) 4067145, (044) 4082881

\begin{abstract}
The article considers issues relating to the system of continuous professional training of aviation specialists and formulates fundamental prerequisites of psychological endurance reliability of aircrews in terms of flight safety problems. Analysis is given of factors affecting the efficiency of the "pilot - airplane" interactive system. Results of forecasting the measure of significance of the human factor in aviation for the next 10-15 years viewed in the context of medical and psychological aspects are presented. The structure of personal individuality developed on the basis of psycho-physiological selection allows the formation of professionally significant qualities in aviation specialists to be influenced and the actions of every crewmember in case of an emergency to be forecast.
\end{abstract}

Keywords: flight safety, continuous education, the human factor, "pilot - airplane" interaction, psycho-physiological factors, professional and psychological selection.

\section{Introduction}

The study of the theoretical and methodological basis of the impact of the human factor on flight safety is made necessary by the need to solve the social and practically significant problem of self-improvement and self-actualization of the creative personality in his/her professional activity.

Present-day society's need for the availability of a capacity to develop the creative personality is practically not met, encountering a wall of failure to comprehend the full importance and urgency of the problem under consideration. The fact that the old problems aggravate and new ones arise - calls for a multi-vector investigation of the problem and proves that we should work on shaping professionals who are better prepared to develop themselves.

The series of air crashes that recently shook the world of aviation was due to either design or manufacturing faults in aircraft adverse weather conditions, collision with other aircraft or birds or being hit by an anti-aircraft missile. In the end, behind every such situation stands a human being who was not conscientious enough while doing his/her job or failed to correctly assess the situation when making a decision 
about performing the flight or making a choice of flight mode.

We can thus speak here of some statistical regularity since the "crew factor" (the crew's inadequate actions) is among the causes of 70-80 percent of air accidents (AA), which points to systemic and organic deficiencies in the system of aircraft operation, irrespective of aircraft type [1].

Experts agree on the significance of the human factor (HF) as the main cause of air accidents and incidents, although no significant progress has been made in developing a uniform approach towards the investigation of the role of man in air accidents. In a broad sense therefore the HF involves all human activities. While the human operator (HO) is the most flexible, adaptable, and important element in the air transport system, at the same time he/she is the most vulnerable element from the point of view of possible adverse effects on his/her activity. The term "pilot error" has no positive implication from the point of view of AA prevention and has the reverse effect since it only makes it possible to identify a failure in the interactive system "crew - aircraft - medium" but not the cause. In addition, the term does not include indirect factors that play a significant role in air accidents.

Air accident investigation reports usually provide information on what occurred and when it occurred but do not provide any account of why it occurred, which allows us to draw a conclusion that the procedures in AA investigations reflect gross psychological ignorance and administrative aggressiveness towards the HF [9].

A break-through in enhancing flight safety is therefore necessary and possible in terms of the human factor. First, this requires a more thorough approach towards the problem of optimizing the interactive system "crew - aircraft - medium - air traffic control (ATC)", which involves a complex interdependence between the pilot's reliability and that of the equipment. According to Gunner K. Falgren, an IATA working group representative, speaking at an ICAO symposium on flight safety issues, the general impression was that 75 percent of air accidents were HF related, while we could also believe that it was due to the HF that the other causes of accidents made up only 25 percent [7].

Therefore, the topicality of the present study is due to the fact that the problem of the theoretical and methodological basis of the impact of the human factor on flight safety has not been sufficiently researched; it is an essential condition for effective professional activity in the present-day market-oriented environment.

The object of our study is flight safety, and the subject is the theoretical and methodological basis of the impact of the human factor on flight safety.

\section{Essential content of the study}

A flight that involves no danger is known to be a social myth. It is impossible to live and work in the sky without any danger. Its level can only be reduced. The chief component of danger in flight is the state of the human psyche. Most accidents occur during controlled flight when the crew enjoys good health and nothing is wrong with the equipment. Unfortunately, specialists in the psychology of flying are not, as a rule, invited to participate in the investigation of air crashes and aviation medical people deal with the problem of the psychophysiological capabilities of a pilot only in terms of medical-flying expertise. We therefore believe that the cause of many of the problems lies in poor professional culture of both those airline managers who are in control of not only aircraft but also the "dark side" of the crewmember subconscious (conceit, egocentrism, greediness), and those who have been selected to make decisions and act completely independently. Hence, the reliability of a human controlling flight should be determined not only strictly by regulated psychophysiological parameters, but also by such inner qualities as the level of intellectual development, independence, creative thought, and an ability for self-development. At present, scientists have gained a large number of clues as to how the HF affects flight safety. The paradox however lies in the fact that there exist some effective documents regulating civil aviation in Ukraine where some provisions are essentially only of declarative nature due to the absence of a mechanism of implementation. For instance, a number of documents require reasonable selection of flying crewmembers by airlines, the only basic requirement being the flying experience of each crewmember - although there is no scientific rationale available of the required minimum standard of flying experience. Similarly, it is recommended that the personal negative traits of the trainees hampering their successful completion of the flying program be assessed, while these same documents provide neither an approximate list, nor complex methods of psychodiagnostics or psycho-correction of the negative personal traits.

The above factors allow the central objective of the study to be formulated: in order to optimize the dynamically changing organic system "crew - aircraftmedium - ATC", it is necessary and sufficient to ensure an adequate level of training to produce creative specialists who are to be professionals of a high sociocultural standard with well-developed communication skills and whose core characteristic should be an inclination to be creative.

The objective set makes it possible to advance some hypotheses that would allow the problem to be solved: flight safety will improve if, when optimizing the interactive system "crew - aircraft- medium - ATC", we are guided by specialists' integrative readiness to be creatively professional in their activities, considering:

- $\quad$ specific psychological features of professional communication skills functions;

- criteria and indicators for assessing the formation of professional qualities;

- streamlining standards and regulations in line with professional direction;

- interrelation between personal and professional development;

- role and situational organization of the training of would-be specialists. 
Although airlines globally dedicated to achieving higher standards of flight safety have developed and applied special programs such as CRM, LOFT, Risk Management, and Threat and Error Management, commercial aircraft accidents continue to occur. Accident statistics over the last 40 years show that mechanical or maintenance-related accidents are only 15 percent of the total, and have been decreasing, while accidents attributable to flight crews are about 65 percent of the total and show little trend towards reduction [1].

AA analysis shows that mistakes made by crewmembers in 80-85 percent of instances are the consequence of:

- professional lapses and unsatisfactory psychophysiological profiles of the crew;

- infringements of flight and workplace discipline;

- substandard qualifications of flight crewmembers.

Let us consider the influence of each of these factors on flight safety.

Section I. The effect of psycho-physiological reliability of aircrews on solving the problem of flight safety

The primary purpose of investigating the aspects related to the human factor is to obtain information on the cause of an aviation accident the sequence of events during the accident and its consequences. Special attention is paid to studying psycho-physiological elements resulting in the causes of many air accidents. Such characteristics as professional - intellectual perception, ability to make decisions, purposefulness, diligence, fatigue, and disability, being very important variables, are frequently hard to identify. Even when they are known, they are difficult to measure and document. Thus, the connection between any detected deviation from the standards and the true causes of air accidents almost always remain at the level of assumptions. Despite all difficulties, ICAO, making use of the accumulated experience of AA investigation and prevention, has developed a number of concepts declaring the idea that the HF, as the most complex kind of activity, is interdisciplinary by nature, the basic distinction of which is that it is of an applied nature and is more likely to have a problematic rather than disciplinary orientation, its conceptual relationship with sciences about man being similar to that between the designing activity and natural sciences. And just as technical applied sciences relate to natural sciences with practical areas of their application, the number of complex techniques and methods grows in the field of human influence on the optimality of interactive systems. The multilateral character of the HF with the overlap of integrated subjects allows us to consider it as a system of scientific knowledge about people in the environment in which they live and work; their interaction with the equipment, standards, rules, environment, and also people among themselves in their overall activity, i.e. by HF we shall understand a set of personal, medical, and biological factors determining optimum conditions of operation of aircraft (A) and ATC.

Medical-psychological forecast of HF in aviation nowadays does not encourage optimism and testifies to the extreme complexity of the problem: aircraft performance in the next 10-15 years will steadily grow, while psycho-physiological characteristics of man will remain practically at the same level; flight factor aggressiveness will increase 1.5-3 times, while the level of human protection will advance 1.3 times at the maximum; aircraft operating conditions will become more complicated - the complexity and duration of flying activity will increase 2.5-3.5 times, while the time for decision-making will decrease 50-70 percent; mental tension and the extent of fatigue will double or triple; social-economic parameters will also change: the cost of training and professional fitness will triple. All this testifies to the fact that expanded aviation technology capabilities, with the increasing influence of adverse factors on the human body not being compensated, will preclude achieving the needed efficiency of the activities of aviation specialists activities, aircraft, and aviation as a whole, and will lower flight safety levels [6].

It is therefore possible to state that the human factor in aviation acts as an economic reality. It is sufficient to point out that 30-40 percent of losses, due to equipment damage are incurred by the state through problems with the professional and psycho-physiological reliability of aircraft crews and flight support personnel.

Recently the HF concept, the essence of which is that the main component of the interactive system " crew - aircraft - environment" is the crew, has finally been generated. The realistic way of increasing flight safety under the present complicated conditions in aviation should therefore be related, on the one hand, to some complex actions at the state level, and on the other hand, to the study of the dynamics of the development of critical situations in professional activity.

The assessment of the interactive system components (crew - aircraft - medium - ATC) separately does not give an idea of the various processes and interactions that characterize it as a whole. As a way of describing the processes of controlling these system elements, information exchange, etc., we shall make use of the SHELL model recommended by ICAO as an extended version of the model "man-machineenvironment" which facilitates the understanding of the HF system [9].

In this work, we shall examine the main component of the SHELL model, namely, "Subject" and the linking "Subject - subject".

Data that are necessary for the examination of this central component of the model may be divided into such categories: physical, psychological, and psychosociological and, besides, if physical factors determine the physical abilities and limitations of the HO, then psycho-physiological factors are related to the individual himself, representing a complicated organism, which consists of a great number of systems. Psychological and psycho-sociological factors of the component determine the things people bring into their work situations as a result of their knowledge, experience, and intellectual abilities. They are: professional training, knowledge, experience and skills of piloting, perception and analysis of information, degree of attention and level of work 
load; individual peculiarities of a person, his mental and emotional state, and mood and attitude to risk perception. Events and stress, as well as relations between crewmembers and with relevant air and ground control services, are also included.

Let us consider a series of psycho-physiological factors that influence the effectiveness of the interactive system "pilot - airplane". One of the factors of the psychological state influencing a pilot's (crew's) work and air accidents examined by us in the present work is the factor related to the slowing-down or acceleration of human internal psychological time.

The analysis of a great number of expert materials obtained in the process of investigation of serious air accidents has shown that in 82 percent of them "the initial factor" of accident development was related to decisions made by the crew and their actions, and only in 18 percent of cases connected with other causes (technical failures etc.) In particular, these are due to failure to attempt a second approach (when it is obviously necessary), landing when meteorological conditions are below minimum, landing by all means when conditions are not suitable for landing, and so on. Besides, it has been established that 48 percent of air accidents take place due to conscious violations of flying and work discipline by crews; in 31 percent of cases these accidents are directly related to the insufficient psycho-emotional stability of crews.

The statistics of serious air accidents have recently remained invariable (80 percent of air accidents are caused by HF), i.e. improvements in engineering do not bring about alterations in human psychology, which is determined by old in-depth reasons.

Usually, in a quiet state a pilot flies a plane as if automatically (optimal piloting), guided, on the whole, by intuitive subconscious psychological processes with some insignificant participation of his controlling mind, which is based on previous experience and knowledge. The mental process is simultaneous in time with the physical process of aircraft movement, i.e. there is practical coordination between the actual (physical) and subjective (mental) flows of processes in time and space. And the mode of the crew's actions lags behind the actual mode of operation of the object by not more than two seconds.

As soon as the pilot begins thinking about the control process (rational mental activity displayed at the level of conscious logical decisions), an increase in the interval between the pilot's mode of action and actual mode of operation of the object becomes greater than 2 seconds. As a result, the mode of perception is fragmented and distorted and lags behind the actual one, as a rule, with a subsequent increase in the time deficit at the final stage of the flight.

In case of emotional mental activity, the mode of a pilot's (crew's) perception quite is far from the actual one, inadmissibly forestalling it and creating a real danger of passing into a reflex (in fact, off-time) mental activity with the most unpredictable consequences.

For all that, switching from an optimal to emotional and rational activity is accompanied with a pendulous (more exactly - along a sweeping helix) alteration of these, i.e. the displayed activity becomes non-rhythmical.

Another factor of psychological state affecting the crew's work and air accidents is the change in the functional state of the pilot in the process of plane piloting at standard stages of flight (taking off, climb, level flight, and landing approach). According to both psycho-physiological criteria and work load level, the investigated stages of flight may be arranged in accordance with growing values of indices: level flight, climb, take off, and landing approach, which experimentally confirm the expediency of using developed behavioral criteria for a psycho-physiological estimation of tension and reliability of operator activity of the pilot, as they do not contradict data in literature about the difficulty of the execution of the inspected flying mission and about the accident rate in aviation due to the HF $[7,10]$.

Analysis of expert material, which included selfestimation of the loading by the pilot (at each inspected flight stage) and expert estimation of piloting performance made by an ATC controller, was conducted based on the concept of system approach to the estimation of the operator's activity on the basis of such psycho-physiological criteria as biological activity (BA), negative emotional aspects (EA) and positive motivational aspects (MA) of behavior, and indices of piloting performance (PP) [7].

The regressive dependencies between physiological mobilization (PM) of the interactive system "pilot airplane," and the possibility of an air accident due to the $\mathrm{HF}$, and the level of "pilot - airplane" system danger, describing AA probability due to the HF, were identified for the inspected flight stages.

With the help of selected indices for each flight stage, the psycho-physiological criterion of the pilot's tension when operating the plane was estimated - the level of physiological mobilization (PM) characterizing the difficulty of piloting - as well as EA and MA of behavior.

The results of studies have shown that:

- by work load level growth for the captain, the flight stages were distributed in the following order: level flight, climb, take off, landing approach (thus, the highest biological activity was recorded in level flight, and the lowest one - at making landing approach);

- by evidence of the negative emotional component the stages were set in the following order: landing approach, take off, climb, and level flight (thus, MA in most cases has a negative direction and its positive gradient is noted only at the landing approach);

- the PM level was increasing from the stage of level flight (at the landing approach it was already positive);

- the functional state of the pilot was in most cases characterized by different levels of functional stress. The lowest level was observed during level flight, and the highest was noted at take off; at the same time, landing approach was characterized by an average extent of overstrain, which caused the lowest, in a comparative sense, level of operational state (OS). The remaining 
stages of flight were distributed in accordance with the growth of OS as follows: take off, climb, and level flight.

In order to analyze the extent of conformity of objective psycho-physiological criteria of an operator's activity with a pilot's subjective judgment about the influence of his workload during piloting on the reliability of operation, the applicable correlation analysis was carried out. Close linear links of workload magnitude have been detected in the implementation of the inspected flight stages, with EA $(r=+0.95)$, MA $(r=+0.95)$, PM $(\mathrm{r}=+0.95)$, and $\mathrm{OS}(\mathrm{r}=-0.95)$. These data allow us to make the following conclusions:

- the higher the level of negative emotionality;

- the stronger the motivational component;

- the higher the level of tension;

- the lower the "pilot - airplane" system operating condition, resulting in the decrease of piloting reliability.

Besides, the correlation analysis has also shown linear links between the AA probability and the PM level $(\Gamma=+0.94)$, negative EA $(\Gamma=+0.91)$, positive MA $(\Gamma=$ $+0.96)$ components of behavior, and the system operation condition $(\Gamma=-0.95)$.

These data allow us to make the following conclusions:

- the errors in piloting resulting in air accidents as an objective evidence of $\mathrm{HO}$ hazard level characterizing AA possibility because of the HF for inspected flight stages are largely caused by a high degree of emotionality and motivation during piloting;

- the indicated factors quite often result in a considerable psycho-physiological overstrain of the pilot or even in exhausting the psycho-physiological resources of his body and, as a consequence, in a decrease in reliability of the operator's activity as a whole;

- the impairment of piloting performance has a negative effect on the operation condition of the "man airplane" system, that, accordingly, entails a growth in the hazard ranking of the piloted airplane, as a whole, and results in a decrease in the reliability of the implementation of the flying mission in terms of the HF.

On the basis of processing the results of average values of piloting difficulty (PM) and functional condition of the "pilot - airplane" system, taking into account the HF (OS) (examining the flight stages, with the help of the regression analysis method), the exponential dependencies between a AA probability and applicable recorded values of OS for the determination of the hazard level of the "pilot - airplane" system with an allowance for the system OS as a whole have been determined, conditioned by piloting performance and psycho-physiological tension of the pilot and applicable values of PM for the determination of the hazard level of the "pilot - airplane" system taking into account only psycho-physiological tension of the pilot.

As it follows from the obtained dependencies, the hazard level of the "pilot - airplane" system and consideration of the HF depend to a greater extent on the OS of the whole system than on physiological expenditures (mobilization of the pilot's body resources) of a pilot, which allows the hazard level of the system as a criterion of psycho-physiological analysis of AA probability to be considered.

Taking into account this assumption and exponential dependencies, we can conclude that AA due to the HF considering the "pilot - airplane" system OS, as a whole, are very likely, provided the system OS coefficient is equal to 0.29 , and it becomes practically impossible with the coefficient equal to 1 .

The variation of the system OS coefficient from 1.0 up to 0.29 encompasses the whole of the admissible, from the point of view of world air statistics, a range of AA probabilities due to the $\mathrm{HF}$, that can, in our opinion, serve as one of preliminary criteria of the ergonomic estimation of the hazard level of the "pilot - airplane" system at the development and test stages of airplanes, as well as in training aircrews.

On the basis of the criteria mentioned, it is possible to draw the conclusion that during piloting it is necessary that the mobilization of pilot's body vital resources during the operator activity does not exceed 80 percent of his psycho-physiological abilities $[6,8]$.

Section II. Psychosocial aspects of FS related to errors committed by aircraft maintenance personnel during aircraft ground servicing

As new, ever more sophisticated aircraft appear, a dialectic jump that results in the appearance of qualitatively new working conditions is observed when a person can not, even when mobilized, succeed in solving the problems assigned to him.

Errors of aircraft maintenance specialists arise from the common traits of their personalities, state of health, the system of their training, common labour organization, living conditions, relationships in the team, etc. According to American data, out of the total number of failures of ground electronic computer facilities, 30 percent is the share for which maintenance workers are responsible because of poor qualifications. The greatest number of errors by maintenance crewmembers occurs during maintenance of the landing gear -34 percent, an engine and its units -18.5 percent, the airplane frame 22.4 percent, the engine oil system - 11.6 percent [7].

The quality of a maintenance system and removing damages depends on many factors and is determined by a number of factors such as interest in the work, morale, time shortage, information overloads, and so on. Besides, a great number of people of various professions and different qualification levels are engaged in the process of operation and maintenance of aviation technical equipment as well as in the process of management. They deal with technical devices and are interrelated among themselves making up groups, teams, and shifts. Coordinated efforts of specialists within such teams also influence the FS degree, work efficiency, economic factors, and the psychological climate within the group.

The analysis of errors (violations) during aviation maintenance operations by maintenance staff has made it possible to formulate the main causes of their occurrence. They are: 
- poor discipline of aircraft maintenance facility staff members - 57.6 percent (among them: 57 percent violation of technical rules; 15.8 percent - improper attachment of fittings; 13.8 percent - improper aircraft towing control and special vehicles approach to the aircraft; 11.9 percent - foreign objects getting into the engines or any other aircraft systems;

- low level of the staff's professional knowledge 25.9 percent among them: 63 percent of errors are closely connected with improper replacement works and 21.8 percent - improper professional knowledge of specialists;

- imperfection of technical documentation, connected with improper maintenance technology and ambiguous instructions in guiding documents.

The true way of increasing FS in the current difficult situation in aviation is, in our view, the following:

- the development of complex measures on the government level (creating a socio-psychological service within aviation divisions), on the one hand;

- turning to more profound research of objective and subjective factor genesis and critical situation development dynamics in the professional activity along with an active use of preventive behavior in the practice of up-to-date psychological knowledge, on the other.

Thus, investigating the correlation of objective and subjective risk factor dynamics, substantiating the existence of zones, which are different in terms of "sensitivity" to the activity break-down (safety zone, risk zone, "augmentation zone" and "danger elimination zone"), permits one to come to the following conclusion:

- safe activity is accomplished only when subjective realization of risk factor levels is greater or equal to the levels of objectively existing danger factors (and it is necessary to take into consideration the different individual significance of disagreement between objective and subjective factors of danger in a risk zone and danger augmentation zone);

- revealed regularity of safe operation can be provided only knowing the dynamics of objective and subjective danger factors in case of a critical situation (an emergency).

Furthermore, one of the main causes of accidents in the socio-technical system is lack of comprehension that a man is a subject of some activity, a subject of vital activity, and a social subject. The practice of forming individual characteristics or activity components, adopted in professional training, does not pay proper attention to integral subject aspects of organizing man's activity and so does not provide full psychological readiness to nonstandard situations.

The above-mentioned allows making the following conclusion:

- control of a human operator's activity may be considered as behavior of a subject in the operating environment, assuming, along with regular functioning, potential danger to himself, other people, and the operated object, and in a critical situation potential danger turns into a real one when the operative environment acquires signs of a "hostile environment" for the operator;
- identification of objective danger factors rating and the measure of adequate subjective notion of them permits safe activity regulations to be to substantiated: subjective perception of a danger factor level must be greater than or equal to the levels of objectively existing danger factors;

- training staff to be psychologically ready for adequate actions in an emergency must be combined, to our mind, with teaching what needs to be done in a situation of increased complexity, "exhausted" abilities and skills of activity that are close to the margins of activity disruption, which can be fulfilled through developing means of individual self-regulation and introduction of vocational psychology into the process of self-regulation in critical situations.

Section III. Profession-oriented selection - as the basis of optimizing the interactive system "crew - A medium - ATC"

It is common knowledge that an aviation working body is a group of people united in their activity by the common task of providing FS, which implies efficiency, regularity, and reliability. The following two groups of aviation personnel take part in solving this problem:

- ground personnel performing timely and qualified pre-flight and post-flight maintenance of the aircraft;

- a flight crew fulfilling their duties in a confined space under dynamically changing conditions of their activity during flight.

Successful solution of these tasks depends on coordination of operations in the teams, each of which has its own socio-psychological peculiarities, the ability to fulfill difficult tasks under the conditions of stringent time limits, economy, safety, flight comfort, and regularity of passenger traffic.

At the same time, each member of the working body possesses his/her individuality, which is an integral property combining his/her congenital and individual characteristics on discovering which one can judge his/her abilities and preferable sphere of activity.

Dynamic characteristics of a person's psychology (emotionality, speed of response, activeness, plasticity, rigidity, and sensibility) are revealed through his/her basic qualities. They form certain behavior and the person's style of activity that appear to be fusion of his/her personal traits, congenital and gained in the process of growing up and socializing. The main driving force of personality development, however, is his/her programmable qualities: purposefulness, intellect, and (self)-consciousness, which are successfully developed at the earliest stages of selection and specialization of a young person's personality. At this very stage, a person's purposefulness comes to life, his/her interests, inclinations, outlook, convictions, "life concept", and behavior motivation. Hence, we can conclude that the problem of a pilot's performance reliability may and should be solved at a very early stage of professional selection for civil aviation training school enrolment.

Much attention is attached to selection in aviation, and, in particular, to psychological selection, in many 
countries of the world. But certain inconsistencies can easily be found in the proposed system of psychological selection based mainly on short-term data assessment obtained from tests. It turns out that the use of this kind of data might be useful in forecasting success in the early stages of flight training but can not help overcome problems of forecasting pilots' professional adaptation and performance [4]. A successful solution to the problem of professional selection is possible by observing certain methodological principles, among which we can identify objectivity, forward-looking orientation, and competence, called to secure an all-round assessment of a prospective trainee's professional suitability. To this end, a system of criteria should be taken into account that includes medical examination data (among them a physical fitness assessment); psychological examination data, data on previous academic achievement and entrance exam results; the moral traits of the applicant, etc. (when selecting for refresher courses, the list should include criteria for assessing professional knowledge, skills, and professional adaptability).

Under "forward-looking orientation" in professional selection, we take to mean not only its orientation towards the closest objectives of professional training and adaptation, but also consideration, while designing a selection procedure, of specific features and requirements of future professional activities and the following stages in the professional career. This requires a personalityoriented approach in considering candidates and is possible through the use of an adequate professionalgraphic study of various activity levels as applied to different aircraft types and flying skill mastery acquisition. The study of personality traits can only be successful on condition of studying the ME of the candidate - peculiarities of the emergence, dynamics, and transformation of activity motivation, representing a complex of methods and methodological problem.

Therefore, the task of professional selection is to identify professionally significant qualities in enrollees needed for a pilot to perform adequately in his/her daily work and in emergencies, i.e. a number of psychological qualities, and specific personality traits that would allow the future cadet to master aircraft piloting and perform operational flying.

In our view, of great importance among the personality traits and conditions essential for successful study and aircraft piloting professional activities are:

- moral qualities (a high level of general culture);

- the level of basic training;

- a profound and sustainable interest in professional activity;

- a wide range of general interests;

- a complex of traits that determine attitudes to work (diligence, being organized, being responsible, etc.);

- logical and quick thinking, rapid response;

- volitional qualities and emotional steadiness;

- a high level of self-control;

- a high level of development of long- and shortterm memory;

- attention switching and concentration, its stability;
- rapidity and accuracy of perception of data obtained from different analyzers (sight, hearing, tactile, etc.) measured in enrollees when providing careerguidance and during medical and psycho-physiological examinations.

Let us focus on the psychological examination of enrollees that, in order to obtain objective and reliable information, should meet a number of standard requirements: appropriate environment and conditions under which it is conducted; methodology and procedure. Here, psychological examination methodologies directed at studying peculiar features of attention, its steadiness, scope distribution, ease of switching, degree of fatigue or lapses in attention, space perception resilience, the ability to perform multi-task activities allow assessing qualities of thinking such as the ability to analyze and compare, to draw logical conclusions; identifying the ease of establishing associative relations; measuring the rate of switching from one type of mental activity to another; studying short-term memory, etc. In addition, in the process of a psychological examination of enrollees, an investigation is made into the specific features of their movement coordination and emotional stability, sensor-motor qualities, specific features of attention and short-term memory, moral qualities and outlook, inclinations, professional orientation, etc. - the factors taken into consideration when enlisting them in flying schools and by instructor-pilots in pilot training to help develop certain professionally significant qualities.

Another problem of professional selection is the need to assess the ability of an individual to act professionally in abnormal situations (emergencies, potential emergencies) - the ability of a person for the socalled "non- standard activity". Candidates to favor in selection are those who are characterized by a desire for constant engagement in work and find recreation in switching to other kinds of activity.

Regarding further improvement of psychological selection, we believe there is a need to develop some theoretical content for the concept of individual emotional maturity, integrating traits and qualities that determine the adequacy of response and stability of psychological functioning under stress. Of importance is the direction in methodology that studies the sociopsychological mobility of an individual, i.e. efficient adjustment to the circumstances of group isolation characterized by forced interpersonal contacts, dynamics in relations, etc. - here considerable attention should be paid to the effectiveness of the involvement of an individual in teamwork and the ability to control processes within a team.

Present-day psycho-psychological selection also features the use of new approaches designed to study the ability for self-regulation in psychic, somatic, and vegetative responses based on research in the field of socalled biological feedback (biofeedback), now successfully being developed in many countries, i.e. the ability to control one's own responses. Methods of controlling some of the parameters such as: galvanic skin response, muscle tone, heartbeat and respiration rate have been developed in this field and are gaining more and 
more acceptance in the practice of psycho-physiological selection to assess the plasticity and stability of processes in higher nervous activity stability and the prospects for teaching a candidate to self-regulate his/her functions.

Flying aircraft now and in the future is and will be performed, largely, not by individual pilots (like in smaller aircraft), but by crews. The very fact of a small team of humans finding themselves under specific flight conditions calls for the elaboration of a number of important problems related to measuring the efficiency of this team's activities, the solution of problems of people's interaction in flight, and the maximum reduction of the stress that arises under flight conditions.

The following conclusions can be drawn from the analysis carried out:

- only by systemic training, taking due account of scientifically grounded methodologies, may the personality structure of an individual be formed;

- the targeted influence on an individual, throughout the process of training, can facilitate the formation of personal qualities required for a particular team of airmen; it can also provide forecasting the behavior of each crew member in an emergency;

- the above mentioned factors could considerably reduce inadequacies in the actions of "air complex" operators and increase flight safety, in the end;

- general differentiating indicators of being inclined towards danger are identified as emotional and temperamental features and a number of traits and qualities indicating a person's being prone to accidents: inattention poor power of observation (discretion), lack of sensor-motor coordination, low endurance, too high (or too low) propensity to risk.

The analysis of air accidents due to flight crews shows that 51.6 percent are the result of behavior related to decision making. Wrong actions are determined in the first place by the dangerous (irrational) quality of professionally intellectual abilities, among which are the following: ignoring, impulsiveness, the feeling of invulnerability, self-assurance, and humbleness. The experience of American researchers shows that correction of dangerous strategies in the process of psychological and professional training permits a considerable increase (up to 20 percent) in FS. But the lack of tests using complex psycho-diagnostics of dangerous positions hampers the timely planning and implementation of corresponding preventive work.

Drawbacks in the system of the professional training of flight crews hamper the process of intensive formation of highly professional pilots. According to the director of the Airbus Industry Consortium center for training flight crews and ground personnel in flight operation, aircraft maintenance and overhaul, the professional training of pilots (PPT) in France (one of the best in the world) provides little or no monitoring of any scientific and technical progress in CA. The HF in the process of professional training is not paid enough attention. During flight training, crewmembers are mainly taught "pure pilotage" techniques, whereas most flight maneuvers on up-to-date aircraft are automatic. At the same time, the matters of general flight leadership and controls of the situation at work places and in the aircraft cockpit in general are practically not considered. Professional training is not concerned with various aspects of human relations, leadership psychology, or pilots' responsibility.

\section{Professional training as the basis of FS}

The fundamental concepts developed by ICAO concerning the human factor, specifying basic types of professional and individual traits of flight crew members as well as maximum possible characteristics of flight crew actions and morale in emergencies were taken as a basis for programs of preventing aviation accidents. This led to forming the characteristics and methods of HF evaluation under different conditions of pilots' professional skills in the process of flight tests and aircraft operation while studying psychological and medical abilities of a personality. An important addition to ICAO documents regulating the concrete content of programs for training aviation specialists was the introduction of a new characteristic, "Flight training", in the 8th edition of these documents, where requirement standards of special knowledge for future specialists were for the first time differentiated in accordance with special subjects. The requirements of studying the abilities and limitations of a human-operator in the flying activity were included in these subjects. These demands have the same status as the knowledge in the field of any other chapter of a traditional course of professional training. It determines the necessity of working out a corresponding program of training and introducing new concepts into the system of this training.

In our view, it is very important that the new edition includes in the section "Skills" the qualitatively probable qualification evaluations of the level of flight crew psychological and professional training. It is required that amateur - pilots of commercial aviation permanently demonstrate their skills of flying aircraft so that accomplishment of a flight is never in serious doubt. The requirement for airline pilots (AP) to demonstrate their skills is extended so that those in possession of an AP certificate are to have demonstrated the ability:

- to make correct decisions and to provide qualified control and flight observation;

- to understand and abide by the rules of coordinating the actions of crew members and the procedure if one of the crew members loses his ability to work;

- to maintain active communication with the other crew members.

Civil aviation flight crew requirements were developed according to ICAO standards. They determine the professional, moral, and socio-psychological characteristics of flight crews, as well as the main principles of assigning and forming crews. The content of the basic requirements for professional training evaluation, however, does not allow the standards to be established to supervise crew resources that would optimize crew activities in the aircraft cockpit in terms of all resources available to ensure flight safety and the corresponding professional training to optimize the mode 
of activities. The analysis of the causes and circumstances of aviation accidents shows the necessity for special professional training of flight crews (PTFC). This necessity allows the formation of habits to detect and identify in a timely manner the signs of dangerous changes in flight conditions and to make the most rational decisions and achieve efficient cooperation among crewmembers to realize them. The common methods of PTFC are oriented, first of all, to working out and consolidating the algorithms of activities specified in the Flight Operation Manual, which does not allow the skills of creative thinking in a non-standard situation to be acquired.

The efforts of air companies and aviation-oriented educational establishments to improve the PTFC system have led to the development of diverse programs and systems of psycho-professional training. Let us consider the most significant ones.

The LOFT Program is a program that is widely used in world civil aviation and is oriented towards active pilots.

CRM is a program designed for active pilots but is concerned with engineering psychology and social psychology of flight crews and larger units $[1,9]$. This program stresses the significance of psycho-professional training, it even becomes a separate component, thus raising the role of the pilots' and crew's basic education. That is, CRM is the program of measures that optimize the crew's activities in the cockpit concerning the use of all possible resources to secure flight safety (FS) and implement optimum modes of activities.

In addition, this program stresses the importance of individual work in which tests and audiocassettes are used. The goal of individual work in the program is to prepare crewmembers for the main seminar, the aim of which is:

- to develop skills to supervise the coordinated work of crew members;

- to master the skills of effective communication;

- to comprehend aircraft crew work in stressful situations and in a state of fatigue;

- to raise awareness of personal potential abilities and the importance of self-improvement.

Interesting results were obtained when United Airlines introduced the program: the number of hours flown per each lost aircraft went up from 1.3 (1970$1979)$ to $4.2(1980-1986) \mathrm{mln}$. hours, i.e. an increase of more than three times.

One-time training within the CRM program cannot give a long-lasting effect, however. A pilot develops his attitudes and standards of professional behavior during the whole period of his flying career, beginning with primary flight training.

Training programs that aim to develop a pilot's ability to make correct decisions at the first stages of primary professional training are of great interest. Worthy of note is the ERAU (Embry Riddle Aeronautical University) manual which is based on the methodology of focusing the cadet's attention on three factors determining his decision-making: the pilot, aircraft and environment. The items relating to the pilot are: the factors of professional training, experience, health, stress, the level of weariness, etc.; the aircraft factors are associated with its performance, airworthiness, condition of functional systems and equipment. The environment factors include meteorological conditions, relief, ATC operation, etc.

Research shows that crewmembers that have gone through fundamental professional training according to the ERAU manual make up to 54 percent fewer mistakes during control flights than those who have not. According to expert evaluations the all-round introduction of the ERAU manual into the practice of PTFC allowed the number of aircraft accidents to be reduced by not less than 5-20 percent.

The analysis of the programs shows that the development of trainees' professional mental abilities is possible only through special purposeful professional training, the success of which is based on trainees' professional and psychological selection (PPS) to be instructed in this specialty and on the development of rational methods of flight training that take into account the conditions of functioning and psycho-physiological abilities of the body.

The positive effect of the introduction of PPS into aviation-oriented educational establishments has been confirmed by convincing data. The following fact was established in a German aviation medical institute: the dismissal of each candidate having low PPS results saves $\$ 150$ thousand; timely PPS in the process of education saves about $\$ 1$ million for each 100 graduates. As mentioned above, in preparatory courses it is also advisable to determine not only the students' professionally relevant qualities, but their intellectual abilities as well - the ability to make decisions, evaluate situations, generate new knowledge and skills, and regulate their behavior accordingly - for the ongoing professional activity.

To identify the indicated qualities of a person, one can use the methodology of revealing interpersonal relationship, which, using the advantages of homothetic and ideographic methods of social and psychological tests and the provisions of the theory of constructs, with the help of the principle of expert evaluation of the qualities of each member of the team and the tests done, allows us:

- to evaluate communication skills;

- to identify the actual leaders of the team tested;

- to evaluate the popularity of each member of the structural sub-unit.

- to form a psychological portrait structure for each person tested according to the test results;

- to design a scheme of interpersonal relations in a structural sub-division according to the analysis of individuality structures and psychological portrait;

- to control the development of a worker's professional skills;

- to predict the liability assessment of each team members' qualities;

- to predict the actions of each team member in an extreme situation.

Moreover, the analysis of social-psychological factors for the purposes of flight crew staffing has shown 
that the type of relations within the team is directly related to their intellect development structure and level (verbal and non-verbal). Taking into account the practical significance of problems of compatibility of an individual and a small group, the socio-psychological crew climate, we should be well advised to include the socio-metric methodology of group assessment of an individual into complex psychological testing that uses the modern approach to individual/small group compatibility diagnostics. The foundation for the methodology could be provided by the use of N.V. Bakharyeva's acceptability scale that employs the "direct" ("I choose") and "reflex" ("I am chosen") criteria [9]. Then, initial assessment according to the first criterion will form a matrix of "actual" acceptability (socio-matrix), and according to the second criterion they will make up "expected" acceptability (auto-socio-matrix), on the basis of which the following socio-metric indicators (indexes) will be calculated: emotional effusiveness (EE), socio-metric status (SS), expected socio-metric status (ESS), and expected emotional effusiveness (EEE). Indexes EE and ESS characterize the attitude of a personality to the group, i.e. the role the personality performs, and indexes SS and EEE are the numerical expression of the group attitude to an individual and indicate the prescribed role of the individual.

The methodology of group assessment of an individual allows not only to estimate the compatibility level of an individual and the group, but to conduct targeted correction of cadets' (students') behavior in flying and academic groups and to evaluate the efficiency of measures taken to optimize the socio-psychological climate in the groups, as well.

The personality dynamic structure concept can serve as the basis for personality control, which includes four interconnected substructures [6]:

- bio-psychical characteristics (age and inherited abilities, instincts and inclinations, the type of nervous system and typological peculiarities of the individual);

- psychical processes (sensation and perception, imagination and reflection, memory and attention, thinking and speech, emotions and will);

- psychosocial formations (knowledge, abilities, skills, habits, aims, values, experience);

- social qualities (character, talent, orientation).

Nowadays we have well-developed methods of diagnosing and correcting personality substructures. Our main task, therefore, is to make up a psycho-professional characteristics model of a certain crewmember and, then, to constantly monitor him (including feedback, i.e. correction) throughout his study at the higher educational establishment [3].

\section{Conclusions}

Research conducted allows us to draw the following conclusion:

- the structure of personal individuality based on psycho-diagnostic and pedagogical methods allows the development of the required individual qualities that are essential to certain groups of airmen to be directly affected during studies. It also allows the actions of each team member in emergencies to be predicted. This significantly reduces the possibility of inadequate actions on the part of aviation complex operators and, in the end, ensures improved flight safety;

- the leader, by knowing the individual qualities of each team member and having the structure of their psychological portraits, could be able to make up an effective and well-organized team capable of working in any situation. Furthermore, the leader can constantly improve the professional qualities of the team created by making the best use of each member's psychological portrait characteristics;

- summing up the above flight safety control elements by the human factor, we are able to form a concept developed from the standpoint of the systemic approach that consists in constant dynamic variation of psycho-professional characteristics of each element of a triad "individual - small group - team" over the course of time as a result of both natural development of control objects and successive effects of the appropriate complex of measures directed at improving flight safety. The possibility of the operational introduction (realization) of any element processed in the framework of the triad into the system of professional training is an important feature of the concept.

The elaborated systemic approach to psychological and professional student training that takes the human factor into account covers nearly all its aspects and elements; namely, the implementation of the approach is accomplished through:

- the identification of psycho-physiological characteristics of "the human operator", which may help to learn the profession of an airman or can be dangerous for flight safety;

- the identification of man's motives to work safe in the interactive system;

- training to identify stress and overcome it;

- developing the ability to estimate danger levels;

- taking into account the social-psychological situation in the flight crew;

- the assessment of skills to make the right (rational) decisions;

- studying the ways to change the behavior of the human-operator.

- Further improvement of the process of psychological and professional training can be achieved along the following lines:

investigation of the paradox of the main psychological dominant and formation of the multivariate function reflecting the "utility-safety" of a crew member as a decision-maker;

- elaboration of an automated system for recognizing potentially "accident-prone" individuals on the basis of results of complex psychological tests using mathematical methods of image discrimination;

- simulation of the pilot's and crew's motives, in general, to work safely using A. Denisov's "information objectives" and "motivation chains"; 
- application of the catastrophe theory mathematical model to develop an educational model in the process of psychological and professional training.

\section{References}

1. Boeing, Statistical Summary Of Jet Airplane Accidents. Worldwide Operations 1959-2002.

2. Kim C.Y. A Study on the Type and Compatibility of Flight Crew Personalities // Journal of the Korean Society for Aeronautical and Flight Operation. 1996, 4 December. - P. 39-62.

3. Malhazov $\boldsymbol{O}$. The Realization Specialties of Computer Technologies in Psycho-physiological Researches in Aviation // Safety in Aviation. Proceedings of the Second World Congress "Aviation in the XXIst Century". - Kiev, 2005. - P. 6.43-6.47.

4. Petrenko A. Transformation of Psychological Criteria of Professional Fitness of Civil Aviation Pilots in the Process of Aircraft Generation Replacement // Ibid. - P. 6.31-6.37.

5. Баевский Р.М., Барсукова Ж.В., Бродягин Н.А. Оценка функционального состояния организма при трудовых нагрузках по показателям активности регуляторных систем. - Челябинск: МЗ РСФСР, 1986. - 17c.

6. Казак В.Н. Облік соціально-психологічних факторів при формуванні екіпажів літальних апаратів // Україна - AGAPO - NATO - Росія. Зб. матеріалів першого міжнародного симпозіуму 3 проблем авіаційної і космічної медицини. - 1997. - С. 271-274.

7. Копанев В.И. Авиационная медицина и безопасность полета // Психофизиологические и медицинские проблемы безопасности полета. Москва: ВИНИТИ, 1989. - С. 3-6.

8. Платонов К.К. Структура и развитие личности. Москва: Наука, 1986. - 256 с.

9. Рева А.Н. Эргономические основы первоначальной подготовки: Дис. ... докт. тех. наук. - Киев: КМУГА. -76 с.

10. Токарев В.И. Роль человеческого фактора в обеспечении безопасности полетов // Проблемы безопасности полетов.- Москва: ВИНИТИ, 1992. - Вып. 5. - С. 28-36.

\section{List of abbreviations}

1. AA - air accident

2. HF - human factor

3. HO - human operator

4. IATA - International Air Transport Association

5. ICAO - International Civil Aviation Organization

6. FS - flight safety

7. A - aircraft

8. ATC - air traffic control

9. CRM - Cockpit Resource Management

10. LOFT - Line-oriented flight training

11. BA - biological activity

12. EA - emotional aspect

13. MA - motivational aspect

14. PM - physiological mobilization

15. OS - operational state

16. PPT - pilots' professional training

17. AMF - aircraft maintenance facility

18. CA - civil aviation

19. AP - airline pilot

20. PTFC - professional training of flight crews

21. ERAU - Embry Riddle Aeronautical University

22. PPS - professional and psychological selection 